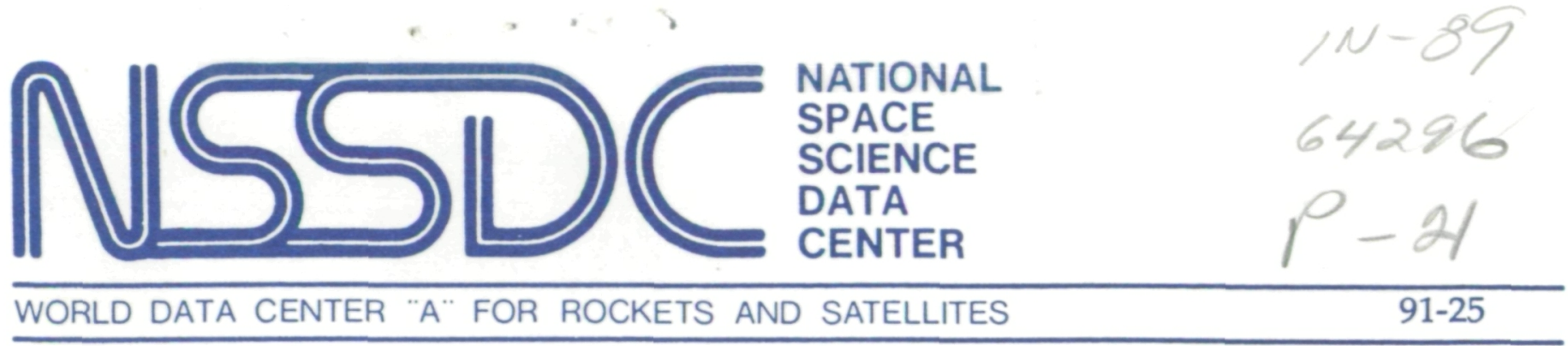

\title{
Radio Sky Mapping from Satellites at Very Low Frequencies
}

L. R. O. Storey 


\title{
Radio Sky Mapping from Satellites at Very Low Frequencies
}

\author{
L. R. O. Storey
}

November 1991

National Space Science Data Center (Code 930.2) NASA/Goddard Space Flight Center Greenbelt, Maryland 20771, U. S. A. 


\section{Contents}

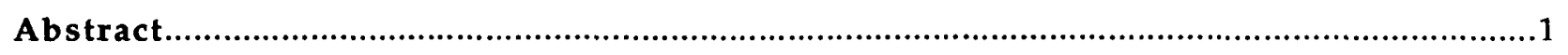

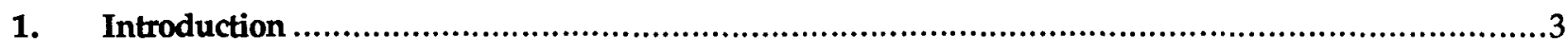

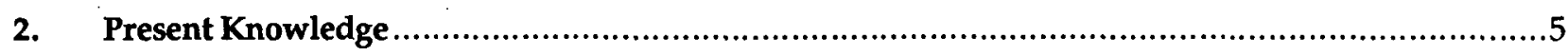

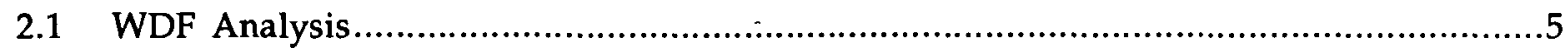

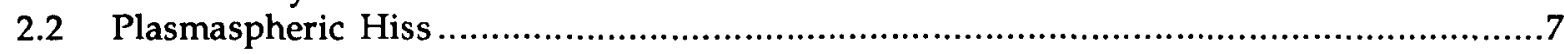

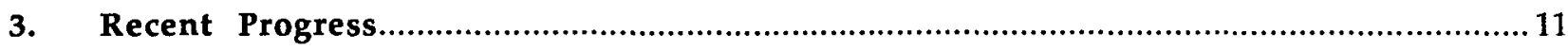

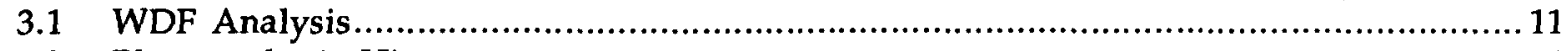

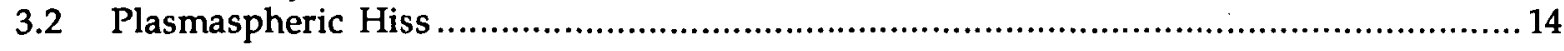

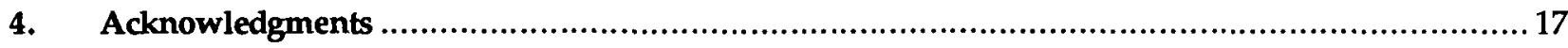

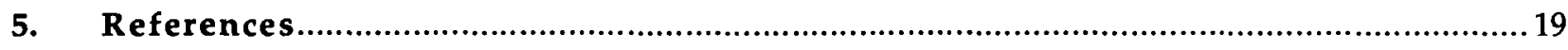

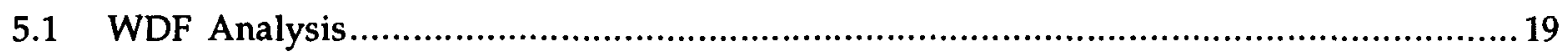

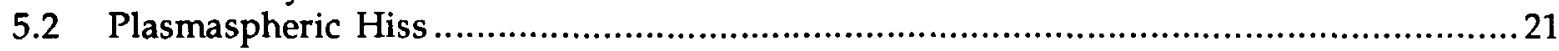

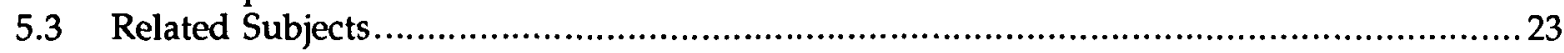




\section{Abstract}

Wave Distribution Function (WDF) analysis is a procedure for making sky maps of the sources of natural electromagnetic waves in space plasmas, given local measurements of some or all of the three magnetic and three electric field components. The work that still needs to be done on this subject includes solving basic methodological problems, translating the solutions into efficient algorithms, and embodying the algorithms in computer software. One important scientific use of WDF analysis is to identify the mode of origin of plasmaspheric hiss. Some of the data from the Japanese satellite Akebono (EXOS D) are likely to be suitable for this purpose. 


\section{Introduction}

WDF analysis is a generalized form of radio direction finding. As input, it accepts in situ data on electromagnetic (EM) plasma waves in space, specifically data in the form of simultaneous measurements of several or all of their six (three electric and three magnetic) field components. Its output consists of sky maps, showing, at a given frequency and for a given EM plasma wave mode, how the intensity of the waves varies as a function of their direction of arrival at the point of observation; this function is the WDF. Its methodology was developed originally in France in the early 1970s by the present author in association with F. Lefeuvre, who had it as a thesis assignment [1-3]. Lefeuvre received his State Doctorate degree from the University of Orléans, France, in 1977 and then continued working in the same area. With his French colleagues, and later in association with some Japanese workers, he analyzed data from the European Space Agency's satellites GEOS 1 and GEOS 2, from the NASA satellite International Sun-Earth Explorer (ISEE) 1, and from the Soviet satellite Aureol 3. The outcome of their sustained efforts is a large body of published work, referenced in $\S 5.1$, which has established the value of WDF analysis for the experimental study of EM wave phenomena in space plasmas.

Plasmaspheric extremely-low-frequency or very-low-frequency (ELF/VLF) hiss is a phenomenon that has been much studied in this way because of its importance in the dynamics of Earth's radiation belts. It occurs widely and frequently and has been observed off and on for a quarter of a century, yet its mode of origin is still not fully understood. Its random, continuous character makes it well suited for WDF analysis.

Section 2 of this report describes the present state of published knowledge about WDF analysis ( $\S 2.1$ ) and plasmaspheric hiss ( $(2.2)$, explaining why further research is needed on both fronts. Briefly, the methodology of WDF analysis needs improvement because at present too many data are being wasted. As for plasmaspheric hiss, the problem of its origin remains unsolved because the right data are not yet available, though some progress is being made with the data in hand.

These topics were investigated by the author at Stanford University from February 1984 to November 1986. Some possible causes of the waste of data were identified, and solutions to this problem found, but time did not then permit the development of new software based upon them; this work, which is described in § 3.1, is now being continued at the National Space Science Data Center (NSSDC). Meanwhile, in a collaboration with Lefeuvre and others, a study has been made of plasmaspheric hiss data from the ISEE 1 satellite, analyzed with existing software. The results from this study and also from some related work performed by R. A. Helliwell and his colleagues at Stanford are described in $\S$ 3.2. Section 3 also lists the improvements that are still needed in the methodology of WDF analysis and explains how it could then be applied to solving the persistent problem of the origin of plasmaspheric hiss. 


\section{Present Knowledge}

\subsection{WDF Analysis}

The aim of methodological research on WDF analysis is to devise procedures for making radio sky maps at VLF with the best possible directional resolution: The difficulty is that the resolution offered by a single antenna is very poor indeed. The resolution is determined by the beam width, and an antenna of characteristic dimension $d$ receiving EM radiation of wavelength $\lambda$ has a beam width of the order of $\lambda / d$, provided that $\lambda \ll d$. However, this condition cannot be met at VLF. At $10 \mathrm{kHz}$, for instance, the free-space wavelength is $30 \mathrm{~km}$, and although the wavelengths in the magnetospheric plasma are substantially smaller, they are still much greater than the dimensions of any antenna that can be deployed from a satellite. Hence all such antennas, whether electric or magnetic, behave like Hertzian dipoles and have the same simple cosine-squared reception pattern, offering little in the way of resolution.

The way around this difficulty is to collect all the available relevant information and make the best possible use of it. This means that, to begin with, accurate measurements should be made of as many as possible of the six components of the EM wave field, namely the three electric components and the three magnetic ones. The static magnetic field vector should be measured as well, together with the properties of the ambient plasma: The electron concentration is essential, the ion composition highly desirable. Theoretical knowledge about the waves, in particular as to how their polarization depends on their direction of propagation, should also be brought to bear. A simple but very helpful fact is that the WDF cannot be negative. Finding out how best to exploit this set of information is the task of methodology.

At the start of any WDF analysis, there is a preliminary step of data reduction. Since the electric and magnetic field data are random, or assumed so, only their statistical properties are of interest. Hence the first step is to use the waveforms of the $N(\leq 6)$ measured components of the EM field to estimate the $N^{2}$ power spectra, i.e., the $N$ purely real auto-spectra together with the real and imaginary parts of the $N(N-1) / 2$ complex cross-spectra, that characterize their second-order statistics; this operation greatly reduces the amount of data. It is convenient to regard these spectra as the elements of a Hermitian $N \times N$ spectral matrix. At any given frequency the $N^{2}$ values of the spectral matrix elements are the actual input data for the WDF analysis.

The earliest work on the methodology of WDF analysis solved the direct problem of how to calculate the spectral matrix, given the WDF $[1,6,7]$. There then remained the inverse problem of estimating the WDF from the spectral matrix, which is much more difficult. 
The initial approach was by model-fitting, which consists of adopting, for the WDF, some analytic model governed by a finite number of adjustable parameters [2]. The unique set of parameter values that fits the data best can be found by nonlinear optimization, using the principle of maximum likelihood. Unfortunately, this simple procedure is unsatisfactory because, in general, there is no way of telling from the data whether or not the model is appropriate.

There is, however, an exception to this rule, which occurs when all of the waves observed at the spacecraft appear to be arriving from just a few discrete directions, typically one or two. Moreover, it is possible to tell from the data whether this state of affairs exists. Only in this special case, which is quite common, has the model-fitting method of WDF analysis so far found practical use [4, 9].

In the general case, the inverse problem is more difficult to solve. The solution favored by Lefeuvre and his colleagues was based on the principle of maximum entropy, which had been used previously to solve similar problems in signal theory, image processing, geophysics, and many other fields. Development of the maximum-entropy method for WDF analysis and some initial application of it to multicomponent ELF/VLF wave data from the GEOS 1 satellite provided Lefeuvre with the material for his thesis [3]. For its large-scale application he was assisted by C. Delannoy, a professional software engineer and author of several books on computer programming. The first description of their method in the open literature was published in 1979 by Lefeuvre and Delannoy jointly [5], and their GEOS 1 data analyses were published two years later [8]. At the same time they undertook to improve the method by experimenting with the solution of analogous inverse problems in one dimension instead of two [10, 11]. Finally, they embodied it in a large Fortran 77 software package called MAXENTWDF, which is now in the public domain [24].

Figure 1 indicates succinctly how the analysis proceeds. The maximum-entropy model is fitted to the input data by an iterative process of nonlinear optimization. Sometimes the iteration fails to converge to a solution, in which case the data are rejected. Even when a solution is obtained, it may have to be rejected because it is too unstable, i.e., too sensitive to small errors in the data. Solutions for the WDF that pass the test for stability can be plotted as sky maps.

Other persons have contributed to the development of WDF analysis. Recently, a Swedish physicist $K$. Rönnmark and his collaborators have refined the definition of the WDF and have shown that its spatio-temporal variations in a weakly inhomogeneous magnetoplasma are governed by a transport equation analogous to the Vlasov equation for particle distribution functions $[29 ; 30]$. Turning their attention to the methodology, they have developed a variant of the maximum-entropy method in which the maximization is performed by "simulated annealing," a process used in statistical mechanics and in operations research $[31,58]$. This work has been undertaken with a view to analyzing data from satellites of the Viking series, in the Swedish national space program.

Of all the satellites designed to make multi-component measurements of ELF/VLF wave fields, the one best equipped for this purpose is the Japanese satellite Akebono (EXOS D), launched in February 1989. The ELF/VLF wave instrument for Akebono was supplied by I. Kimura. It measures three components of the wave magnetic field and two components of the wave electric field, in the frequency range from 100 $\mathrm{Hz}$ to $12.7 \mathrm{kHz}$. The electric components are sensed by two crossed dipole antennas, each $60 \mathrm{~m}$ tip-to-tip. A unique feature of the instrument is that it can measure the vector impedances of the electric antennas; these data are used to correct for the significant attenuation occurring between the antennas and the preamplifiers, thus improving the accuracy of the electric field measurements. A number of satellites now being built will also be equipped for multi-component ELF/VLF wave measurements. In scheduled order of launch, they include the Auroral Probe satellite in the Soviet INTERBALL program, the four Cluster satellites in the International Solar-Terrestrial Physics (ISTP) program, and the Polar satellite in the NASA Global Geospace Science (GGS) program. The persons responsible for the multicomponent wave measurements in these three programs are F. Lefeuvre, N. Cornilleau-Wehrlin, and D. A. Gurnett, respectively. 


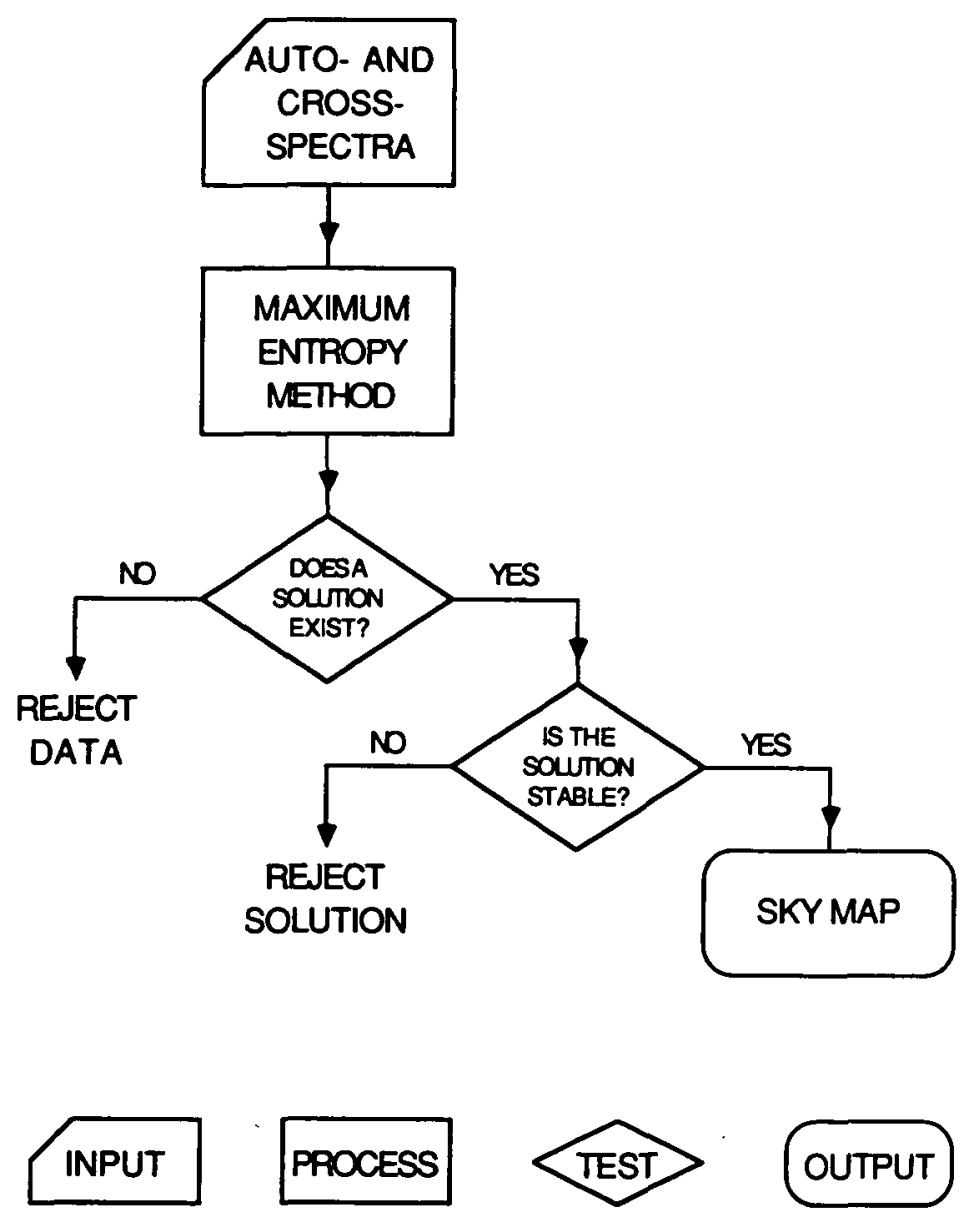

Figure 1. Existing Maximum-Entropy Method of WDF Analysis.

Maximum-entropy WDF analysis of wave data from past or current satellites has already provided the subject matter for a number of papers, of which Lefeuvre and his colleagues are the principal authors $[3,8,12,14,17-19,22,23]$. They have also written several papers in collaboration with a Japanese group at the University of Nagoya [16, 20, 21, 25-27] and have collaborated briefly with U.S. $[14,18]$ and Soviet [19] workers. The next sub-section reports the impact that some of this work has had on current understanding of plasmaspheric hiss.

\subsection{Plasmaspheric Hiss}

The ELF/VLF emission known as plasmaspheric hiss is a widespread and almost permanent feature of the natural EM wave field in Earth's plasmasphere $[38,39]$. It is noise-like, seeming structureless when displayed on a frequency-time spectrogram, although at times it is associated with structured wave phenomena such as chorus. It covers a band of frequencies from a few hundred hertz to a few kilohertz. 
The upper cut-off frequency is always well below the local electron gyrofrequency [36, 37, 42]. On the other hand, most of the hiss band is above the local proton gyrofrequency, in a frequency range where only the whistler mode exists. Thus plasmaspheric hiss consists of EM waves propagated in the whistler mode.

Plasmaspheric hiss is widely believed to be produced by the Doppler-shifted electron gyroresonance instability (or whistler instability for short), the immediate source of free energy being the energetic (tens of $\mathrm{keV}$ ) electrons trapped in Earth's radiation belts; the anisotropy of their distribution in pitch angle makes the whistler mode unstable [34]. The theoretical growth rate of the waves is largest in the magnetic equatorial plane, just below the plasmapause, so this region is often presumed to be their source, from which they spread out to fill the plasmasphere.

The first comprehensive theory of the origin of plasmaspheric hiss was put forward by Kennel and Petschek (K \& P) in a classic paper that has influenced all subsequent thinking in the field $[35,40]$. They recognized that the whistler instability is convective, not absolute, which means that although the unstable region around the magnetic equator can amplify whistler-mode waves that traverse it, such waves cannot be generated there spontaneously. Moreover, they estimated that the amplification resulting from a single pass through this region is insufficient to raise the waves from their thermal level to the levels observed for the hiss. Multiple passes are needed, which would occur by propagation back and forth between the two hemispheres along the magnetic lines of force, with reflections from the ionosphere or lower magnetosphere at either end. This process of feedback by propagation would convert the amplifying region into an oscillatory one, generating waves at levels limited by quasilinear modification of the distribution function of the energetic electrons. In sum, according to $K \& P$, the hiss is produced by the plasmasphere acting as a laser.

The main difficulty with this theory concerns the direction of propagation of the waves (i.e., the wave normal direction), which governs their spatial growth rate in the amplifying region around the magnetic equator. $K \& P$ found that the waves propagating parallel to the magnetic field ("longitudinally") are amplified the most, but that the amplification decreases as the angle between the wave normal and the field increases, and beyond a certain angle the waves are damped. Now, the wave normal angle varies along the propagation path of a wave, and the way in which it does so can be discovered by ray tracing. Such studies show that, in the plasmasphere, waves starting from the equator with their normals parallel to the field and following paths of the type pictured by $K \& P$ usually return to the equator with their normals oblique. For these waves, at any rate, there can be no further amplification, so no laser action.

Two consequences follow. Firstly, if K \& P's theory is to survive, it must be supplemented by some mechanism for bringing the waves back repeatedly to their source region at the equator with their normals always more or less parallel to the field. Secondly, for testing this or any other theory, it is important to be able to measure wave normal directions in situations where several sets of waves are present at once, all propagating in different directions, hence the value of WDF analysis.

As regards the first of these two points, various mechanisms have been proposed, including guidance of the waves by trapping inside field-aligned ducts $[19,20,41,52,55]$, guidance by propagation just below the plasmapause $[22,56]$, and propagation on closed "cyclic trajectories" with one reflection from the plasmapause in each hemisphere [43]. Of the papers that deal with trapping or guidance, [55] and [56] concern theories of these modes of propagation, while the others present experimental evidence for their roles in producing some kinds of hiss. In $[19,20]$ and also in [22], the evidence comes from WDF analysis: Hiss waves have been observed in ducts or just below the plasmapause with their normals more or less parallel to the magnetic field. Hiss produced as guided waves can also be observed on the ground, because such waves are able to escape from the ionosphere. However, ELF hiss is detected more often in the plasmasphere than it is on the ground, suggesting that its waves are not always guided; this suggestion is borne out by the fact that WDF analyses of plasmaspheric hiss generally show it to be propagating obliquely [21]. The paper [43] on cyclic trajectories is purely theoretical, but some 
relevant experimental results are described in $\S 3.2$. In these three mechanisms, the waves are all supposed to propagate on closed paths.

Several variants of the $K \& P$ theory have been suggested in which the requirement for closed paths is abandoned. Thus Church and Thorne [45], and also Huang and Goertz [46, 47], have traced ray paths for non-ducted waves and have found cases where the waves make several successive passes through the equator with their normals remaining fairly close to the magnetic field, though becoming oblique on later passes. Thus the waves gain more energy than they could on a single pass, but they are not amplified indefinitely; that is, there is no laser action. Church and Thorne suggest that plasmaspheric hiss is produced on such trajectories, by amplification of waves from some non-thermal "embryonic source," which they have not yet clearly identified. Huang and Goertz disagree, having made less favorable assumptions about the distribution function of the energetic electrons responsible for the whistler instability, and there, for the moment, this matter rests.

Recently, Thorne and Summers have considered energetic electron distribution functions that permit the growth of obliquely propagating whistler-mode waves, as well as of longitudinally propagating ones [60]. Their findings may support Church and Thorne's theory of the origin of plasmaspheric hiss, but the application has not yet been made, at least, not to the present author's knowledge.

Using particle as well as wave data from the GEOS 1 and GEOS 2 satellites, Solomon et al. [50] have found cases in which the measured distribution functions of the energetic electrons implied growth rates sufficient to raise ELF waves from thermal level to the observed hiss levels on a single pass through the magnetic equator, as had already been suggested by Cornilleau-Wehrlin et al. [48]. Their data were taken when the satellite was near the equator and just below the plasmapause. They cite WDF analyses by Parrot and Lefeuvre [22], which showed that waves observed at such points were propagating almost parallel to the magnetic field, in agreement with the theory. They recognize, however, that their findings in these cases need not apply to others, and in particular, not to waves observed at places well below the plasmapause; GEOS 1 did not descend below $L=4.5$, while GEOS 2 was in a geostationary orbit. Sky maps of plasmaspheric hiss at lower altitudes show that oblique propagation is more common than parallel propagation, even at points close to the magnetic equator (see § 3.2).

Another common feature of plasmaspheric hiss is that the sky maps often show one - or, more commonly, two - distinct peaks. The occurrence of a peak suggests that the satellite is observing waves from a spatially localized but remote source. The presence of two peaks can be explained by the peculiar relationship that applies between the wave normal and the ray directions in the whistler mode. Two different wave normal directions may correspond to the same ray direction, the latter being the direction of propagation of the energy. Hence, between a localized source and a remote point of observation there may be two propagation paths, corresponding to different wave normal directions. The observed directions are generally consistent with this interpretation.

The current status of the various theories of the origin of plasmaspheric hiss will be summarized in $\S$ 3.2. First, however, the results from some recent research at Stanford University will be presented. 


\section{Recent Progress}

\subsection{WDF Analysis}

Section 2.1 described the maximum-entropy method of WDF analysis in its standard form, which was used for the wave data analyses referred to in $\S 2.2$. It was also used for those studied in the course of the recent work that will be discussed briefly in $\S 3.2$ below.

Unfortunately, experience with WDF analysis has revealed one serious drawback, namely that of wasting data. Not all of the data encountered in practice can be exploited by this method. With some data sets, when their analysis is attempted, either no solution for the WDF can be found, or the solution is unstable. For instance, in a collection of ELF hiss data from GEOS 1, comprising 262 sets of fourcomponent wave field measurements, 171 (65\% of the total) were exploitable if only the three magnetic components were analyzed, but the number fell to $22(8.4 \%)$ if the single electric field component was included in the analysis. Now, it is important to be able to include at least one electric component, because with only the magnetic components the results are ambiguous: There is no way to distinguish between two waves traveling in opposite directions. On the other hand, a wastage rate exceeding $90 \%$ is obviously unacceptable.

Some effort had gone previously towards solving this problem, and one cause of instability had been recognized, namely redundancy in the data. The elements of the spectral matrix are related to the WDF by a set of linear integral equations, and the kernels of these various equations are not altogether linearly independent of one another. The resulting instability can be cured, at the price of some loss of data, by orthogonalizing the kernels and then eliminating some of the smallest ones $[3,5,24]$. The cause of the frequent failures to find any solution at all for the WDF was not known, however.

Therefore, in the methodological work performed at Stanford in 1984-1986, one of the first issues examined was whether solutions for the WDF always exist. Firstly, considering that the WDF cannot be negative, it was found that measurement errors can indeed lead to situations where there are no nonnegative solutions. Secondly, when the data are on the border of the range of values within which such solutions do exist, these solutions are usually both singular and unique, in which case they represent waves arriving from a few discrete directions only. Thirdly, when the data are just inside this border, the maximum-entropy solution is highly unstable in some respects, but not in others; this is an additional cause of instability, distinct from the one just mentioned. These findings, most of which have been published [32], are the bases for the new method of WDF analysis now under development at NSSDC. 


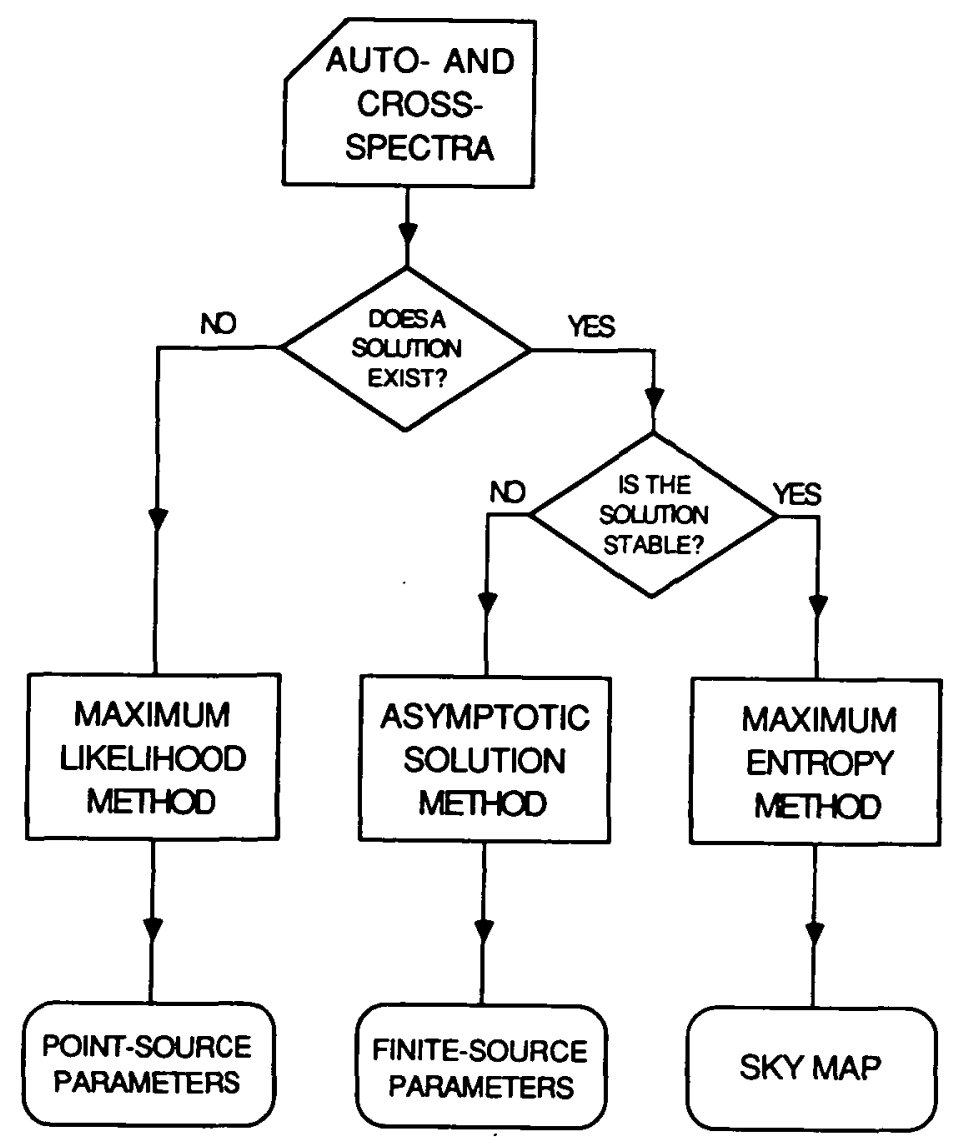

FIgure 2. Proposed New Method of WDF Analysis.

Figure 2 illustrates this method in outline. The main difference from the current method (Figure 1) is that tests for the existence and stability of solutions are applied to the data before these are analyzed, and the route that the analysis follows depends on the outcome.

Two algorithms for performing existence tests have already been devised, the first of which is systematic and yields unequivocal answers, though at the price of much computation [32]. The second, based on work by Agmon et al. [57], involves a random search and is more efficient computationally, but the answers that it yields are only highly probable, not absolutely certain. Future efforts will be devoted to finding an even better algorithm for this purpose.

The role of the existence test is to determine whether any solution for the WDF exists when the data are taken at their face value. If the answer is no, however, this means that the data are erroneous rather than that no solution can be found. In this case, they must be corrected to the most likely set of feasible values, which usually corresponds to a singular solution. Thus, in fact, this test determines whether the solution to be found is singular or non-singular. 
The role of the stability test is to determine, in cases where a solution exists, how close the data are to the border of the region of "data space" within which there is no solution. If they are quite close, then the solution is likely to be unstable.

In Figure 2, the right-hand branch represents the procedure to be followed when the tests indicate that a stable non-singular solution exists. The solution is found by using the principle of maximum entropy. Here the new method differs from the existing one only in points of detail, such as the exact form of the entropy functional.

The central branch in Figure 2 represents the procedure in the case where the data lie close to the border referred to above, and hence are unstable, at least when judged by the stability criteria that previous workers have used. In point of fact, these solutions consist of one or more narrow peaks, and though their heights are unstable (i.e., sensitive to slight changes in the data), their positions and the volumes under them are stable. Moreover, the sub-region of data space within which there is instability, close to the border, is also the domain of validity of certain analytic approximations to the true maximum-entropy solutions; these approximations become asymptotically exact as the data set approaches the border. Hence the proper tactic for dealing with unstable maximum-entropy solutions, which cause trouble for any iterative procedures, is to replace them by the corresponding asymptotic approximations; it is hoped that the data needed for calculating these approximations can be obtained as by-products of the existence test.

Finally, the left-hand branch in Figure 2 represents the procedure to be followed in the case where the outcome of this test is negative, meaning that the data are erroneous and should be replaced by their most likely true values. These are the nearest values, in the maximum-likelihood sense, for which a solution for the WDF exists. Provided that the errors have reasonable statistics, this set of data must necessarily lie on the border, which, as already stated, means that the solution must be singular, consisting of one or several Dirac distributions (or "delta functions"). In physical terms, this means that the wave field consists of just a few discrete sets of waves propagating in well-defined directions, a special case that was discussed previously in \$2.1. Although such singular solutions can be found as limiting cases of the asymptotic approximations mentioned above, a much simpler and more efficient way of finding them is model-fitting: The solution for the WDF is modeled as the sum of the appropriate number of delta functions, the strengths and positions of which are the parameters of the model. Again, preliminary estimates of the values of these parameters should be obtainable from the existence test. With these features based on the principle of maximum likelihood, the new method of WDF analysis will unify the existing maximum-entropy and model-fitting methods $[8,9]$.

The question might be asked as to why no method of this kind has yet been developed in any other of the many fields where linear inverse problems are solved by invoking the maximum-entropy principle. The answer lies in a peculiarity of natural whistler-mode waves in Earth's magnetosphere, namely that WDFs consisting of one or two fairly narrow peaks are quite common, as was mentioned in $\S 2.2$. Such data lie close to the border of the region of data space in which solutions for the WDF exist, so the solutions that they yield are unstable, and moreover, measurement errors can readily displace the data into the region where no solutions exist. In other fields, similar difficulties do not appear because the unknown functions to be solved for rarely have this peaky character. One field in which the unknown functions are sometimes peaky and where the new method may therefore be needed is the mapping of starspots [59]. 


\subsection{Plasmaspheric Hiss}

In the course of the research at Stanford, no analyses of satellite data on plasmaspheric hiss were made by the new method described above, since its development had only just begun. However, in collaboration with Lefeuvre and others, efforts were made to interpret some data from ISEE 1 that he had analyzed by his existing method. A brief account of this work is given in a report prepared for the sponsor [28]. Some of its broader implications, which were not covered in this report, are discussed below.

Since some theories of the origin of plasmaspheric hiss assign an important role to the plasmapause, as a guide or as a reflector for the whistler-mode waves, it was decided to analyze data taken at times following long periods of magnetic quiet, when either there was no plasmapause or it was located unusually far from the Earth, at $L>7$. Nonetheless, strong hiss was observed on all of the four satellite passes from which the data were taken. On two of them, during which the satellite covered large ranges of $L$ while remaining close to the magnetic equatorial plane, the upper cut-off frequency of the hiss increased steadily with decreasing $L$, as several previous authors have reported; at the lowest $L$ values, it was about $10 \mathrm{kHz}$. Waves of these frequencies could not have originated in the region just below the plasmapause, since the electron gyrofrequency, which is the upper limit for propagation in the whistler mode, is only about $2.5 \mathrm{kHz}$ at $L=7$. These observations support the view that plasmaspheric hiss is generated over a wide range of $L$-values and not only just below the plasmapause.

Confirmation of this view was forthcoming from the WDF analyses, which on one of the two equatorial passes yielded evidence for a broad source region extending over the range $2.4<L<4.3$. Though near to the equator, most of the waves observed on these passes were propagating obliquely; similar results have been reported recently by Sonwalkar and Inan, based on data from the Dynamics Explorer 1 satellite [51]. In the ISEE 1 data, however, some waves were also observed at relatively small wave normal angles, and a ray-tracing study suggested that they might have been propagating on cyclic trajectories as proposed by Thorne et al. [43].

These results, taken with those published by previous experimenters, have led the present author to the following conclusions concerning the mode of origin of plasmaspheric hiss. The generation mechanisms proposed by Kennel and Petschek [35], by Thorne et al. [43], and by Solomon et al. [50], among others (see $\$ 2.2$ ), are all physically plausible and can act whenever the necessary conditions exist, in which case they give rise to waves that cross the magnetic equator with their normals at small angles to the magnetic field. However, hiss occurs even when the conditions for none of these mechanisms exist, and then it appears to be generated near the equatorial plane over a wide range of $L$ values, with the wave normals at large angles to the field. It would be premature to speculate as to what the generation mechanism may be in such cases. Suffice it to say that the present author shares the opinions of certain other workers who, having noted that hiss often occurs in association with the more coherent emission known as "chorus," have suggested that the two may be produced by the same basic mechanism, which must be a nonlinear rather than a quasilinear one [44, 49, 51, 53]; the recent observations by Sonwalkar and Inan [54] of hiss emissions triggered by lightning-generated whistlers are supporting evidence.

It is now clear what experimental data are required for identifying this unknown mechanism. The need is for multi-component ELF/VLF wave data, including accurate measurements of at least two and preferably all three electric field components so that the WDFs can be derived without ambiguity. Moreover, these data should be taken by a satellite in a polar orbit with its perigee in the plasmasphere. Then any equatorial region in which waves are being generated should be readily detectable, from the reversal that would occur in the predominant direction of the wave energy flux as the satellite passed through it. Other aspects of the variation of the WDF through such a region should help to identify the generation mechanism: for example, for those waves that have their 
Poynting vectors parallel or anti-parallel to the spacecraft orbit at the equator, it should be possible to determine the spatial growth rates. Suitable data are already being taken by the Japanese satellite Akebono, and, if all goes well, several other satellites will be doing the same in a few years from now. 


\section{Acknowledgments}

The work at Stanford University described in $\$ 3.1$ was supported by Grant No. ATM-8318186 from the National Science Foundation. This report was written while the author held a National Research Council Senior Research Associateship at NASA Goddard Space Flight Center. The study of plasmaspheric hiss summarized in $\S 3.2$ was made in association with F. Lefeuvre, M. Parrot, L. Cairó, and R. R. Anderson; a fuller account of it can be found in the November 1991 issue of the Journal of Geophysical Research.

This report will also appear in Environmental and Space Electromagnetics (proceedings of the URSI Commission E Symposium, Tokyo, September 4-6, 1989), edited by H. Kikuchi, in April 1992. 


\section{References}

In each of the three following sub-sections, the references are given in approximate chronological order.

\subsection{WDF Analysis}

1. Storey, L. R. O., and F. Lefeuvre. "Theory for the Interpretation of Measurements of a Random Electromagnetic Wave Field in Space." Space Research 14, 381-386, 1974.

2. Lefeuvre, F., and L. R. O. Storey. The Analysis of 6-Component Measurements of a Random Electromagnetic Wave Field in a Magnetoplasma: - Model Identification. Technical Report No. CRPE/41, CNRS Center for Research in Physics of the Environment, 1977.

3. Lefeuvre, F. Analyse de Champs d'Ondes Electromagnétiques Aléatoires Observés dans la Magnétosphère à Partir de la Mesure Simultanée de leurs Six Composantes (Analysis of Random Electromagnetic Wave Fields in the Magnetosphere, Based on Simultaneous Measurements of Their Six Components). State Doctorate Thesis, University of Orléans, France, 1977.

4. Buchalet, L. J. Analyse de Champs d'Ondes Electromagnétiques à Partir de Modèles à une ou deux Directions Privilegiées (Analysis of Electromagnetic Wave Fields, Based on Models with One or Two Privileged Directions). Third Cycle Thesis, University of Orléans, France, 1979.

5. Lefeuvre, F., and C. Delannoy. "Analysis of a Random Electromagnetic Wave Field by a Maximum Entropy Method." Ann. Télécomm. 34, 204-213, 1979.

6. Storey, L. R. O., and F. Lefeuvre. "The Analysis of 6-Component Measurements of a Random Electromagnetic Wave Field in a Magnetoplasma: - I. The Direct Problem." Geophys. J. R. Astron. Soc. 56, 255-270, 1979.

7. Storey, L. R. O., and F. Lefeuvre. "The Analysis of 6-Component Measurements of a Random Electromagnetic Wave Field in a Magnetoplasma: - 2. The Integration Kernels." Geophys. J. $R$. Astron. Soc. 62, 173-194, 1980.

8. Lefeuvre, F., M. Parrot, and C. Delannoy. "Wave Distribution Functions Estimation of VLF Electromagnetic Waves Observed on Board GEOS 1." J. Geophys. Res. 86, 2359-2375, 1981.

9. Buchalet, L. J., and F. Lefeuvre. "One and Two Direction Models for VLF Electromagnetic Waves Observed on Board GEOS 1." J. Geophys. Res. 86, 2377-2383, 1981. 
10. Delannoy, C., and F. Lefeuvre. Logiciel de Résolution d'un Problème Inverse à 1 Variable: - 1. Exposé des Méthodes (Software for the Solution of an Inverse Problem with 1 Variable: - 1. Description of the Methods). Technical Report No. CRPE/80, CNRS Center for Research in Physics of the Environment, 1981.

11. Delannoy, C., and F. Lefeuvre. Logiciel de Résolution d'un Problème Inverse à 1 Variable: - 2. Documentation d'Utilisation (Software for the Solution of an Inverse Problem with 1 Variable: - 2. Users' Guide). Technical Report No. CRPE/81, CNRS Center for Research in Physics of the Environment, 1981.

12. Lefeuvre, F., T. Neubert, and M. Parrot. "Wave Normal Directions and Wave Distribution Functions for Ground-Based Transmitter Signals Observed on GEOS 1." J. Geophys. Res. 87, 6203$6217,1982$.

13. Lefeuvre, F., and D. Lagoutte. "Bias for Spectral Density Estimates of Electromagnetic Wave Field Components in a Magnetoplasma." Ann. Geophys. 1, 265-270, 1983.

14. Lefeuvre, F., M. Parrot, L. R. O. Storey, and R. R. Anderson. Wave Distribution Functions for Plasmaspheric Hiss Observed on Board ISEE 1. Technical Report No. LPCE/6, CNRS Laboratory for Physics and Chemistry of the Environment, 1983.

15. Storey, L. R. O. "The Energy Density of Electromagnetic Waves in a Cold Magnetoplasma." J. Plasma Phys. 32, 309-318, 1984.

16. Hayakawa, M., Y. Yamanaka, M. Parrot, and F. Lefeuvre. "The Wave Normals of Magnetospheric Chorus Emissions Observed on Board GEOS 2." J. Geophys. Res. 89, 2811-2821, 1984.

17. I agoutte, D., and F. Lefeuvre. "Multispectral Analysis for Electromagnetic Wave Field Components in a Magnetoplasma: Application to Narrow-Band VLF Emissions." J. Geophys. Res. $90,4117-4127,1985$.

18. Lefeuvre, F., and R. A. Helliwell. "Characterization of the Sources of VLF Hiss and Chorus Observed on GEOS 1." J. Geophys. Res. 90, 6419-6438, 1985.

19. Beghin, C., J. C. Cerisier, J. L. Rauch, J. J. Berthelier, F. Lefeuvre, R. Debrie, O. A. Maltseva, and N. I. Massevitch. "Experimental Evidence of Field-Aligned ELF Plasma Ducts in the Ionospheric Trough and in the Auroral Zone," Résultats du Projet ARCAD 3 et des Programmes Récents en Physique de la Magnétosphère et de l'Ionosphère (Results from the ARCAD 3 Project and Recent Programs in Magnetospheric and Ionospheric Physics). Toulouse, France, Cepadues Editions, $517-$ 527, 1985.

20. Hayakawa, M., N. Ohmi, M. Parrot, and F. Lefeuvre. "Direction Finding of ELF Hiss Emissions in a Detached Plasma Region of the Magnetosphere." J. Geophys. Res. 91, 135-141, 1986.

21. Hayakawa, M., M. Parrot, and F. Lefeuvre. "The Wave Normals of ELF Hiss Emissions Observed Onboard GEOS 1 at the Equatorial and Off-Equatorial Regions of the Plasmasphere." J. Geophys. Res. 91, 7899-7999, 1986.

22. Parrot, M., and F. Lefeuvre. "Statistical Study of the Propagation Characteristics of ELF Hiss Observed on GEOS 1, Inside and Outside the Plasmapause." Ann. Geophys. 4, 363-384, 1986.

23. Cairó, L., and F. Lefeuvre. "Localization of Sources of ELF/VLF Hiss Observed in the Magnetosphere: Three-Dimensional Ray-Tracing." J. Geophys. Res. 91, 4352-4364, 1986. 
24. Delannoy, C., and F. Lefeuvre. "MAXENTWDF - A Computer Program for the Maximum Entropy Estimation of a Wave Distribution Function." Comput. Phys. Comm. 40, 389-419, 1986.

25. Hayakawa, M., M. Parrot, and F. Lefeuvre. "The Wave Distribution Functions of Plasmaspheric ELF Hiss: GEOS 1 Observation in the Equatorial Region." Mem. Natl. Inst. Polar Res. (Japan), Spec. Issue 47, 157-172, 1987.

26. Hayakawa, M. "The Generation Mechanism of ELF Hiss in Detached Plasma Regions of the Magnetosphere, as Based on the Direction Finding Results." Mem. Natl. Inst. Polar Res. (Japan), Spec. Issue 47, 173-182, 1987.

27. Muto, H., M. Hayakawa, M. Parrot, and F. Lefeuvre. "Direction Finding of Half-Gyro-Frequency VLF Emissions in the Off-Equatorial Region of the Magnetosphere and Their Generation and Propagation." J. Geophys. Res. 92, 7538-7550, 1987.

28. Storey, L. R. O. Final Project Report on Wave Distribution Function Analysis of Plasmaspheric Hiss (Prepared under NSF Grant No. ATM-8318186). Technical Report No. D-711-2, Space, Telecommunications, and Radioscience Laboratory, Stanford University, 1987.

29. Rönnmark, K. "The Evolution of Spectral Densities in Weakly Inhomogeneous Plasmas." $]$. Geophys. Res. 92, 11,053-11,058, 1987.

30. Rönnmark, K., and J. Larsson. "Local Spectra and Wave Distribution Functions." J. Geophys. Res 93, 1809-1815, 1988.

31. Oscarsson, T. E., and K. G. Rönnmark. "Reconstruction of Wave Distribution Functions in Warm Plasmas." J. Geophys. Res. 94, 2417-2428, 1989.

32. Storey, L. R. O., and S. Chapron. "Conditions for the Existence and Uniqueness of Solutions to a Constrained Linear Inverse Problem." Inverse Problems 4, 249-271, 1988.

33. Hayakawa, M., K. Hattori, S. Shimakura, M. Parrot, and F. Lefeuvre. "Direction Finding of Chorus Emissions in the Outer Magnetosphere and Their Generation and Propagation." Planet. Space Sci. 38, 135-143, 1990.

\subsection{Plasmaspheric Hiss}

34. Cornwall, J. M. "Cyclotron Instabilities and Electromagnetic Emission in the Ultra-Low and Very Low Frequency Ranges." J. Geophys. Res. 70, 61-69, 1965.

35. Kennel, C. F., and H. E. Petschek. "Limit on Stably Trapped Particle Fluxes." J. Geophys. Res. 71, $1-28,1966$.

36. Dunckel, N., and R. A. Helliwell. "Whistler-Mode Emissions on the OGO 1 Satellite." J. Geophys. Res. 74, 6371-6385, 1969.

37. Russell, C. T., R. E. Holzer, and E. J. Smith. "OGO 3 Observations of ELF Noise in the Magnetosphere: 1 - Spatial Extent and Frequency of Occurrence." J. Geophys. Res. 74, 755-777, 1969. 
38. Russell, C. T., R. L. McPherron, and P. J. Coleman. "Fluctuating Magnetic Fields in the Magnetosphere: 1 - ELF and VLF Fluctuations." Space Sci. Rev. 12, 810-856, 1972.

39. Thorne, R. M., E. J. Smith, R. J. Burton, and R. E. Holzer. "Plasmaspheric Hiss." J. Geophys. Res. 78, 1581-1596, 1973.

40. Etcheto, J., R. Gendrin, J. Solomon, and A. Roux. "A Self-Consistent Theory of Magnetospheric ELF Hiss." J. Geophys. Res. 78, 8150-8166, 1973.

41. Chan, K. W., and R. E. Holzer. "ELF Hiss Associated with Plasma Density Enhancements in the Outer Magnetosphere." J. Geophys. Res. 81, 2267-2274, 1974.

42. Parady, B. K., D. D. Eberlein, J. A. Marvin, W. W. L. Taylor, and L. J. Cahill, Jr. "Plasmaspheric Hiss Observations in the Evening and Afternoon Quadrants." J. Geophys. Res. 80, 2183-2198, 1975.

43. Thorne, R. M., S. R. Church, and D. J. Gorney. "On the Origin of Plasmaspheric Hiss: The Importance of Wave Propagation and the Plasmapause." J. Geophys. Res. 84, 5241-5247, 1979.

44. Koons, H. C., "The Role of Hiss in Magnetospheric Chorus Emissions." J. Geophys. Res. 86, 6745$6754,1981$.

45. Church, S. R., and R. M. Thorne. "On the Origin of Plasmaspheric Hiss: Ray Path Integrated Amplification." J. Geophys. Res. 88, 7941-7957, 1983.

46. Huang, C. Y., and C. K. Goertz. "Ray-Tracing Studies and Path-Integrated Gains of ELF Unducted Whistler-Mode Waves in the Earth's Magnetosphere." J. Geophys. Res. 88, 6181-6187, 1983.

47. Huang, C. Y., C. K. Goertz, and R. R. Anderson. "A Theoretical Study of Plasmaspheric Hiss Generation." J. Geophys. Res. 88, 7927-7940, 1983.

48. Cornilleau-Wehrlin, N., J. Solomon, A. Korth, and G. Kremser. "Experimental Study of the Relationship Between Energetic Electrons and ELF Waves Observed on Board GEOS: A Support to Quasilinear Theory." J. Geophys. Res. 90, 4141-4154, 1985.

49. Helliwell, R. A., D. L. Carpenter, U. S. Inan, and J. P. Katsufrakis. "Generation of Band-Limited VLF Noise Using the Siple Transmitter: A Model for Magnetospheric Hiss." J. Geophys. Res. 91, 4381-4392, 1986.

50. Solomon, J., N. Cornilleau-Wehrlin, A. Korth, and G. Kremser. "An Experimental Study of ELF/VLF Hiss Generation in the Earth's Magnetosphere." J. Geophys. Res. 93, 1839-1847, 1988.

51. Sonwalkar, V. S., and U. S. Inan. "Wave Normal Direction and Spectral Properties of Whistler Mode Hiss Observed on the DE 1 Satellite." J. Geophys. Res. 93, 7493-7514, 1988.

52. Koons, H. C. "Observations of Large-Amplitude, Whistler Mode Wave Ducts in the Outer Plasmasphere." J. Geophys. Res. 94, 15,393-15,397, 1989.

53. Sonwalkar, V. S., and U. S. Inan. "Lightning as an Embryonic Source of VLF Hiss." J. Geophys. Res. 94, 6986-6994, 1989. 


\subsection{Related Subjects}

54. Smith, R. L. "Propagation Characteristics of Whistlers Trapped in Field-Aligned Columns of Enhanced Ionization." J. Geophys. Res. 66, 3669-3707, 1961.

55. Inan, U. S., and T. F. Bell. "The Plasmapause as a Wave Guide." J. Geophys. Res. 82, 2819-2827, 1977.

56. Agmon, N., Y. Alhassid, and R. D. Levine. "An Algorithm for Finding the Distribution of Maximal Entropy." J. Comput. Phys. 30, 250-258, 1979.

57. Kirkpatrick, S., C. D. Gelatt, Jr., and M. P. Vecchi. "Optimization by Simulated Annealing." Science 220, 671-680, 1983.

58. Sonwalkar, V. S., and U. S. Inan. "Measurements of Siple Transmitter Signals on the DE 1 Satellite: Wave Normal Direction and Antenna Effective Length." J. Geophys. Res. 91, 154-164, 1986.

59. Cameron, A. C., and K. D. Horne. "Maximum Entropy Reconstruction of Starspot Distributions," Cool Stars, Stellar Systems, and the Sun, eds. M. Zeilik and D. M. Gibson, 205-208, 1986.

60. Thorne, R. M., and D. Summers. "On the Marginal Stability Criterion for a Loss-Cone Distribution." Ann. Geophys. 6, 275-286, 1988. 